PROCEEDINGS OF THE

AMERICAN MATHEMATICAL SOCIETY

Volume 126, Number 12, December 1998, Pages 3461-3465

S $0002-9939(98) 05021-7$

\title{
ORDER COMPLEXES OF NONCOMPLEMENTED LATTICES ARE NONEVASIVE
}

\author{
DMITRY N. KOZLOV
}

(Communicated by Jeffry Kahn)

\begin{abstract}
We prove the following theorem: Let $\mathcal{L}$ be a finite lattice, $x \in \overline{\mathcal{L}}$. Assume $B$ is a set of elements of $\mathcal{L}$ which includes all complements of $x$ and is included in the set of all upper (lower) semicomplements of $x$. Then $\Delta(\overline{\mathcal{L} \backslash B})$ is nonevasive, in particular collapsible. This generalizes results of several previous papers, where, in different generalities, it has been proved that the mentioned complex is contractible.
\end{abstract}

\section{INTRODUCTION}

The study of topological properties of noncomplemented lattices may be said to have been triggered by a paper of H.H. Crapo [Cr]. Namely, as a trivial corollary of his celebrated complementation formula [Cr, Theorem 3], one can derive that the Möbius function of a noncomplemented lattice is zero. On the other hand, the reduced Euler characteristic of an order complex of a poset (see Definition 2.1) is equal to the Möbius function evaluated on that poset. Hence, the conversion of Crapo's result into algebraic topology language gives: the reduced Euler characteristic of the order complex of a noncomplemented lattice is zero.

This was later strengthened by K. Baclawski, who showed in [Ba, Corollary 6.3] that the order complex of a finite noncomplemented lattice is $\mathbb{Z}$-acyclic (here and in the rest of the paper we often identify the poset with its order complex). Although Baclawski's approach was brilliant, using the Leray spectral sequence in an intricate way, it was far from being combinatorial and only yielded acyclicity.

A further improvement was made by A. Björner (see [B81, Theorem 3.3]), where he succeeded in showing that a noncomplemented lattice is contractible. Also this proof used topology machinery, such as the Nerve Lemma. In the same year, in a more general situation, the poset was proved to be $\mathbb{Q}$-acyclic by Björner and Baclawski [BB, Corollary 4]. Later in a joint paper of A. Björner and J.W. Walker a more precise result, known as the homotopy complementation formula [BW, Theorem 1.1], was given, enlightening the subject considerably.

The purpose of this paper is to explain the phenomena discovered in [Ba, B81, B94, BB, BW] in a purely combinatorial way. Namely, we shall prove that the simplicial complex associated to a lattice with a certain set of elements removed is nonevasive, in particular collapsible. Noncomplemented lattices are contained in the class of posets for which our result holds.

Received by the editors February 25, 1997.

1991 Mathematics Subject Classification. Primary 05E99, 06A09, 06B99.

(C)1998 American Mathematical Society 
The above mentioned result of A. Björner combined with a theorem that can be found in $[\mathrm{Co}]$, allowed one to conclude that order complexes of noncomplemented lattices can be reduced to a point by a sequence of collapses and anticollapses. The results of this paper reveal that this reduction can actually be performed, avoiding anticollapses.

\section{THE THEOREM}

Throughout the rest of this paper the symbol $\Delta$ will denote an abstract simplicial complex, that is, a subset of the set $2^{[n]}$ such that $\{i\} \in \Delta$, for $i \in[n]$, and if both $X \in \Delta$ and $Y \subset X$, then $Y \in \Delta$. We will adapt the usual notions of the theory of abstract simplicial complexes, such as $\mathrm{lk}_{\Delta}(\sigma), \mathrm{dl}_{\Delta}(\sigma)$, where $\sigma \in \Delta$. For their description see for example [B95, (9.9)].

Let $P$ be a finite partially ordered set, shortly - a poset. We say that $P$ is bounded if it contains largest and smallest elements, which we denote $\hat{1}$ and $\hat{0}$. All the posets in this paper are finite and bounded. We write $\bar{P}=P \backslash\{\hat{0}, \hat{1}\}$. For $x \in P$ we denote $P_{\leq x}=\{y \in P \mid y \leq x\}$ and $P_{\geq x}=\{y \in P \mid y \geq x\}$.

The main objective of this paper is to study topological objects associated to posets, as given by the following definition.

Definition 2.1. Given a finite bounded poset $P$, the order complex $\Delta(\bar{P})$ is the simplicial complex on the vertex set $\bar{P}$ whose $k$-faces are the $k$-chains in $\bar{P}$.

This construction is, of course, just a special case of the classifying space of a category [Qu, Se].

For $x \in \bar{P}$, we have identities $\operatorname{lk}_{\Delta(\bar{P})}(x)=\Delta\left(\overline{P_{\leq x}} \oplus \overline{P_{\geq x}}\right)$ and $\operatorname{dl}_{\Delta(\bar{P})}(x)=$ $\Delta(\overline{P \backslash\{x\}})$; here $\oplus$ denotes the ordinal sum of posets (see [St, Section 3.2] for its definition).

The symbol $\mathcal{L}$ will always denote a finite bounded lattice. For $x \in \overline{\mathcal{L}}$ we write $\mathcal{C} o_{\mathcal{L}}(x)$ for the set of complements of $x$, i.e. the set $\{y \in \overline{\mathcal{L}} \mid x \wedge y=\hat{0}$ and $x \vee y=\hat{1}\}$; while $\mathcal{C} o_{\mathcal{L}}^{\vee}(x)$ will denote the set of upper semicompliments: $\{y \in$ $\overline{\mathcal{L}} \mid x \vee y=\hat{1}\}$.

Definition 2.2. A simplicial complex $\Delta$ is called collapsible if it can be reduced to a single point by a sequence of elementary collapse steps. An elementary collapse step is a replacement of $\Delta$ by another simplicial complex $\Delta^{\prime}=\Delta \backslash\{\sigma, \tau\}$, where $\sigma, \tau \in \Delta$ and $\sigma$ is a proper face of exactly one simplex, namely $\tau$.

See for example [B95, Section 11] for a survey of main results on collapsibility.

Definition 2.3. We say that a simplicial complex $\Delta$ on a finite number of vertices is nonevasive if either $\Delta$ consists of only one vertex or there exists a vertex $x \in \Delta$ such that both $\mathrm{dl}_{\Delta}(x)$ and $\mathrm{lk}_{\Delta}(x)$ are nonevasive.

There exist several equivalent definitions of nonevasive simplicial complexes in the literature; the one above is taken from [B95, (11.1)]. Originally, nonevasive complexes were defined by Kahn, Saks and Sturtevant in [KSS], in order to model the notion of argument complexity. A good survey on the properties of nonevasiveness can also be found there. For example it is proved in [KSS, Proposition 1] that nonevasiveness implies collapsibility and that the implication is strict. Another fact which follows at once from this approach is that a cone is always nonevasive.

Now we present the main theorem of this paper. 
Theorem 2.4. Let $\mathcal{L}$ be a finite lattice. Let $x \in \overline{\mathcal{L}}$ and let $P=\mathcal{L} \backslash B$, where $\mathcal{C}_{\mathcal{L}}^{\vee}(x) \supseteq B \supseteq \mathcal{C}_{\mathcal{L}}(x)$. Then $\Delta(\bar{P})$ is nonevasive, in particular it is collapsible.

Remark 1. A particularly interesting special case is when $B=\mathcal{C}_{\mathcal{L}}(x)=\emptyset$, that is: when $x$ has no complements, then $\Delta(\overline{\mathcal{L}})$ is nonevasive.

Remark 2. For symmetry reasons, the theorem remains true if upper semicomplements are replaced by lower semicomplements.

Remark 3 . The result of Theorem 2.4 can be translated to algorithmic language in the following way.

Let $P$ be as in the formulation of Theorem 2.4. Assume $\mathcal{A}$ is a subset of $\bar{P}$ which is not known in advance. One is allowed to ask questions of the type: "Is $y$ in $\mathcal{A}$ ?", where $y \in \bar{P}$. Then there exists a strategy which in at most $|\bar{P}|-1$ questions determines whether the set $\mathcal{A}$ is a chain in $\bar{P}$.

See [KSS, Section 3] for more detailed description of the interplay of topological and algorithmic properties of (non-)evasiveness.

Proof of Theorem 2.4. We use induction on the number of elements in the poset $\bar{P}$. Since $x \in \bar{P}$, we know that $\Delta(\bar{P})$ is not empty. Furthermore, if $\bar{P}$ consists of only one element, then $\Delta(\bar{P})$ is a simplicial complex consisting of only one point, and hence is nonevasive by Definition 2.3.

Assume now that $|\bar{P}|>1$. In order to show that $\Delta(\bar{P})$ is nonevasive, we shall find a suitable element $y \in \bar{P}$, for which we shall prove the following two claims:

Claim 1. $\Delta(\overline{P \backslash\{y\}})$ is nonevasive.

Claim 2. $\Delta\left(\overline{P_{\leq y}} \oplus \overline{P_{\geq y}}\right)$ is nonevasive.

One way to show that $\Delta(Q)$ is nonevasive (for $Q$ taken from the claims above) will be to write $Q$ in the form $\mathcal{L}^{\prime} \backslash B^{\prime}$, where $\mathcal{L}^{\prime}$ is some lattice, such that $\left|\mathcal{L}^{\prime}\right|<|\mathcal{L}|$, and $\mathcal{C o}_{\mathcal{L}^{\prime}}^{\vee}(z) \supseteq B^{\prime} \supseteq \mathcal{C}_{\mathcal{L}^{\prime}}(z)$, for some $z \in \mathcal{L}^{\prime} \backslash B^{\prime}$, and to use induction.

We divide the main part of the proof into two cases, depending on the choice of $y$.

Case 1. There exists an element $y$, such that $y$ is an atom of $P$ and $x \nsupseteq y$.

Proof of Claim 1. Since $y$ is an atom, $\mathcal{L}^{\prime}=\mathcal{L} \backslash\{y\}$ is a lattice. Also it is clear that $x \in \mathcal{L}^{\prime}$.

Set $B^{\prime}=B$. If $t \in \mathcal{C}_{\mathcal{L}^{\prime}}(x)$, then $x \vee t=\hat{1}$ and $x \wedge t=\hat{0}$ in $\mathcal{L}^{\prime}$. However, the identity $x \wedge t=y$ is impossible in $\mathcal{L}$, since then it would imply $y \leq x$ which we assumed to be false. Hence $x \wedge t=\hat{0}$ in $\mathcal{L}$, so $t \in \mathcal{C}_{o}(x)$ and therefore $B^{\prime} \supseteq \mathcal{C}_{\mathcal{L}^{\prime}}(x)$. Furthermore, if $t \in \mathcal{C} o_{\mathcal{L}}^{\vee}(x), t \neq y$, then $t \in \mathcal{C}_{\mathcal{L}^{\prime}}^{\vee}(x)$, i.e. $\mathcal{C}_{\mathcal{L}^{\prime}}^{\vee}(x) \supseteq \mathcal{C} o_{\mathcal{L}}^{\vee}(x) \cap \mathcal{L}^{\prime}$.

So we have shown that $\mathcal{C}_{\mathcal{L}^{\prime}}^{\vee}(x) \supseteq B^{\prime} \supseteq \mathcal{C}_{\mathcal{L}^{\prime}}(x)$.

Proof of Claim 2. $\mathcal{L}^{\prime}=[y, \hat{1}]$ is a lattice since it is an interval in the lattice $\mathcal{L}$. Let $z=x \vee y$. Since $y$ is an atom and $y \not \leq x$, we know that $x \wedge y=\hat{0}$. On the other hand $y \notin \mathcal{C} o_{\mathcal{L}}(x)$, so $z \neq \hat{1}$.

Set $B^{\prime}=\mathcal{L}^{\prime} \cap B$. First we show that $\mathcal{C}_{\mathcal{L}^{\prime}}(z) \subseteq \mathcal{C}_{\mathcal{L}}(x) \cap \mathcal{L}^{\prime}$. Take $t \in \mathcal{C}_{\mathcal{L}^{\prime}}(z)$. Then $t \wedge z=y$ and $t \vee z=\hat{1}$. But

$$
\hat{1}=t \vee z=t \vee(x \vee y)=(t \vee y) \vee x=t \vee x .
$$






Let $a=t \wedge x$. Clearly

$$
z=x \vee y \geq x \geq x \wedge t=a
$$

and

$$
t \geq x \wedge t=a,
$$

hence

$$
y=t \wedge z \geq a .
$$

Since $x \geq a$, we get $\hat{0}=x \wedge y \geq a$ hence $a=\hat{0}$ and so $t \in \mathcal{C}_{\mathcal{L}}(x) \cap \mathcal{L}^{\prime}$. Now,

$$
\mathcal{C}_{\mathcal{L}^{\prime}}(z) \subseteq \mathcal{C}_{\mathcal{L}}(x) \cap \mathcal{L}^{\prime} \subseteq B \cap \mathcal{L}^{\prime}=B^{\prime}
$$

and

$$
B^{\prime}=B \cap \mathcal{L}^{\prime} \subseteq \mathcal{C} o_{\mathcal{L}}^{\vee}(x) \cap \mathcal{L}^{\prime} \subseteq \mathcal{C} o_{\mathcal{L}^{\prime}}^{\vee}(z) .
$$

To show the last inclusion just note that if $t \vee x=\hat{1}$, then $t \vee z=t \vee x \vee y=\hat{1} \vee y=\hat{1}$.

Case 2. For every atom $y \in P$ we have $x \geq y$.

Let $\tilde{x}$ be any coatom of $P$, such that $\tilde{x} \geq x$. We can simply replace $x$ by $\tilde{x}$, since $\mathcal{C}_{o_{\mathcal{L}}^{\vee}}^{\vee}(\tilde{x}) \supseteq \mathcal{C}_{o_{\mathcal{L}}^{\vee}}^{\vee}(x)$ and $\mathcal{C}_{\mathcal{L}}(\tilde{x})=\mathcal{C}_{\mathcal{L}}(x)=\emptyset$; that is, without loss of generality, we can assume that $x$ is a coatom of $P$. Clearly $\mathcal{C} o_{\mathcal{L}}^{\vee}(x)=\mathcal{L} \backslash\left(\mathcal{L}_{\leq x} \cup\{\hat{1}\}\right)$.

Let $C=\mathcal{C}_{\mathcal{L}}^{\vee}(x) \backslash B$; then $\bar{P}=\left(\mathcal{L}_{\leq x} \backslash\{\hat{0}\}\right) \cup C$. We finish the proof of Case 2 with an easy induction argument. We use induction on $|C|$. If $|C|=0$, then $\bar{P}=\mathcal{L}_{\leq x} \backslash\{\hat{0}\}$, hence $\Delta(\bar{P})$ is a cone and therefore nonevasive. Assume now that $|C| \geq 1$ and let $y$ be one of the minimal elements of $C$. Set $P^{\prime}=\overline{P \backslash\{y\}}$ and $P^{\prime \prime}=\overline{P_{\leq y}} \oplus \overline{P_{\geq y}} . \Delta\left(P^{\prime}\right)$ is nonevasive by the induction hypothesis and $\Delta\left(P^{\prime \prime}\right)$ is a cone with apex $x \wedge y$, hence is nonevasive as well. So, by definition, $\Delta(\bar{P})$ is nonevasive.

We would like to conclude this paper with the following comment. Recall the following theorem proved by Björner and Walker.

Theorem 2.5 ([BW, Theorem 3.2]). Let $P$ be a poset having an element s such that the following two conditions are satisfied:

1. $s \vee x$ or $s \wedge x$ exists for all $x \in P$;

2. if $x<y, s \wedge x$ does not exist, but $s \wedge y$ does exist, then $(s \wedge y) \vee x$ exists.

Then $\Delta(P)$ is contractible. 
Based on the evidence provided by this theorem, we make the following conjecture.

Conjecture 2.6. Under the conditions of Theorem 2.5 the complex $\Delta(P)$ is nonevasive.

\section{ACKNOWLEDGMENTS}

I would like to thank A. Björner, E.-M. Feichtner and G.M. Ziegler for the careful reading of this paper and the anonymous referee for pointing out inconsistencies in the earlier version.

\section{REFERENCES}

[Ba] K. Baclawski, Galois connections and the Leray spectral sequence, Advances in Math. 25, (1977), pp. 191-215. MR 57:9805

[BB] K. Baclawski, A. Björner, Fixed points and complements in finite lattices, J. Comb. Theory, Ser. A 30, (1981), pp. 335-338. MR 82f:06008

[B81] A. Björner, Homotopy type of posets and lattice complementation, J. Comb. Theory, Ser. A 30, (1981), pp. 90-100. MR 82k:06001

[B94] A. Björner, A general homotopy complementation formula, Discrete Math., to appear, preprint 1994, 7 pages.

[B95] A. Björner, Topological Methods, in "Handbook of Combinatorics" (eds. R. Graham, M. Grötschel and L. Lovász), North-Holland, 1995, pp. 1819-1872. MR 96m:52012

[BW] A. Björner, J.W. Walker, A homotopy complementation formula for partially ordered sets, European J. Combin. 4, (1983), pp. 11-19. MR 84f:06003

[Co] M.M. Cohen, A Course in Simple-Homotopy Theory, Springer, New York, 1973. MR 50:14762

[Cr] H.H. Crapo, The Möbius function of a lattice, J. Comb. Theory 1, (1966), pp. 126-131. MR 33:1240

[KSS] J. Kahn, M. Saks and D. Sturtevant, A topological approach to evasiveness, Combinatorica 4, pp. 297-306, 1984. MR 86f:05117

[Mu J.R. Munkres, Elements of Algebraic Topology, Addison-Wesley Publishing Company, Menlo Park, CA, 1984. MR 85m:55001

[Qu] D. Quillen, Higher algebraic K-theory I, Lecture Notes in Mathematics 341, (1973), pp. 85148, Springer-Verlag. MR 49:2895

[Se] G. Segal, Classifying spaces and spectral sequences, Inst. Hautes Études Sci. Publ. Math. No 34, (1968), pp. 105-112. MR 38:718

[St] R.P. Stanley, Enumerative Combinatorics, vol. I, Wadsworth, Belmont, CA, 1986. MR 87j:05003

Department of Mathematics, 2-392, Massachusetts Institute of Technology, 77 Massachusetts Avenue, Cambridge, Massachusetts 02139

E-mail address: kozlov@math.mit.edu, kozlov@math.kth.se

Current address: Institute for Advanced Study, Olden Lane, Princeton, New Jersey 08540 